\title{
Effect of magnesium supplements on serum C-reactive protein: a systematic review and meta-analysis
}

Mohsen Mazidi ${ }^{1,2}$, Peyman Rezaie ${ }^{3}$, Maciej Banach ${ }^{4-6}$, on behalf of Lipid

and Blood Pressure Meta-analysis Collaboration (LBPMC) Group

${ }^{1}$ Key State Laboratory of Molecular Developmental Biology, Institute of Genetics
and Developmental Biology, Chinese Academy of Sciences, Chaoyang, Beijing, China
${ }^{2}$ Institute of Genetics and Developmental Biology, International College, University
of Chinese Academy of Science (IC-UCAS), West Beichen Road, Chaoyang, China
${ }^{3}$ Biochemistry and Nutrition Research Center, School of Medicine, Mashhad University
of Medical Science, Mashhad, Iran
${ }^{4}$ Department of Hypertension, Chair of Nephrology and Hypertension,
Medical University of Lodz, Poland
${ }^{5}$ Polish Mother's Memorial Hospital Research Institute (PMMHRI), Lodz, Poland
${ }^{6}$ Cardiovascular Research Centre, University of Zielona Gora, Zielona Gora, Poland

Submitted: 10 August 2017

Accepted: 18 August 2017

Arch Med Sci 2018; 14, 4: 707-716

DOI: https://doi.org/10.5114/aoms.2018.75719

Copyright @ 2018 Termedia \& Banach

\section{Abstract}

Introduction: The aim of the study was to undertake a systematic review and meta-analysis of prospective studies to determine the effect of magnesium $(\mathrm{Mg})$ supplementation on C-reactive protein (CRP). Design: Systematic review and meta-analysis of randomised controlled trials (RCTs).

Material and methods: Data sources: PubMed-Medline, Web of Science, Cochrane Database, and Google Scholar databases were searched (up until December 2016). Eligibility criteria: Randomized controlled trials evaluating the impact of $\mathrm{Mg}$ supplementation on CRP. We used random effects models meta-analysis for quantitative data synthesis. For sensitivity analysis was used the leave-one-out method. Heterogeneity was quantitatively assessed using the $I^{2}$ index. Main outcome: Level of CRP after Mg supplementation.

Results: From a total of 96 entries identified via searches, eight studies were included in the final selection. The meta-analysis indicated a significant reduction in serum CRP concentrations following Mg supplementation (weighted mean difference (WMD) $-1.33 \mathrm{mg} / \mathrm{l}$; $95 \% \mathrm{Cl}$ : -2.63 to -0.02 , heterogeneity $\left.p<0.123 ; l^{2}=29.1 \%\right)$. The WMD for interleukin 6 was $-0.16 \mathrm{pg} / \mathrm{dl}$ (95\% Cl: -3.52 to 3.26 , heterogeneity $p=0.802 ; l^{2}=2.3 \%$ ), and $0.61 \mathrm{mg} / \mathrm{dl}$ ( $95 \% \mathrm{Cl}:-2.72$ to $1.48, p=0.182$, heterogeneity $p=0.742 ; l^{2}=6.1 \%$ ) for fasting blood glucose. These findings were robust in sensitivity analyses. Random-effects meta-regression revealed that changes in serum CRP levels were independent of the dosage of $\mathrm{Mg}$ supplementation (slope: -0.004 ; $95 \% \mathrm{Cl}:-0.03,0.02 ; p=0.720$ ) or duration of follow-up (slope: -0.06 ; $95 \% \mathrm{Cl}:-0.37,0.24 ; p=0.681)$.

Conclusions: This meta-analysis suggests that Mg supplementation significantly reduces serum CRP level. RCTs with a larger sample size and a longer follow-up period should be considered for future investigations to give an unequivocal answer.

Key words: meta-analysis, magnesium, C-reactive protein.

\author{
Corresponding author: \\ Mohsen Mazidi \\ Key State Laboratory \\ of Molecular \\ Developmental Biology \\ Institute of Genetics \\ and Developmental Biology \\ Chinese Academy \\ of Sciences \\ Chaoyang, Beijing \\ China \\ Phone: +8613167518660 \\ E-mail: moshen@genetics. \\ ac.cn.
}




\section{Introduction}

Magnesium (Mg), as one of the most abundant minerals in the body, is essential for good health. The main food sources of $\mathrm{Mg}$ are whole grains, legumes, nuts, and green leafy vegetables [1]. More recent evidence indicates that dietary intake of $\mathrm{Mg}$ has an impact on several metabolic and inflammatory disorders including hypertension [2], type 2 diabetes [3], metabolic syndrome [4], insulin resistance [5] and cardiovascular diseases [1,6]. Magnesium deficiency, either from inadequate intake, excess excretion or altered homeostasis, is often suspected to be associated with the initiation of many symptoms and diseases [7]. A growing body of evidence supports the important role of magnesium deficiency in the synthesis and release of pro-inflammatory cytokines and acute phase functions, in the impairment of peripheral insulin action, and in the progress of glucose metabolism disturbances $[8,9]$. It has been proposed that the anti-inflammatory response of $\mathrm{Mg}$ may contribute to the beneficial effects on reducing the levels of C-reactive protein (CRP) - a well-known indicator of acute or chronic inflammation [10]. In this regard, one study has reported an inverse relationship between dietary magnesium intake and CRP levels in non-diabetic non-hypertensive obese individuals [11]. However, another cross-sectional study did not find an association between dietary Mg intake and CRP levels [12]. While these findings suggest that magnesium plays a role in the inflammatory response, single studies to date have been limited by sample size, research design and subject traits (gender, ethnicity, age, etc.), and underpowered to achieve a comprehensive and reliable conclusion. On the other hand, dietary supplementation with $\mathrm{Mg}$ with different dosage and duration can have different effects on some indices of inflammatory and anti-inflammatory indexes. Meta-analysis has the benefit of overcoming this limitation by increasing the sample size. Hence, to better understand this issue, the present study aimed to resolve this uncertainty by systematically reviewing the literature and performing a meta-analysis of all randomized control trials investigating the effects of Mg supplementation on serum CRP levels.

\section{Material and methods}

We followed the guidelines of the 2009 Preferred Reporting Items for Systematic Reviews and Meta-Analysis (PRISMA) statement [13, 14]. Due to the study design (meta-analysis) neither Institutional Review Board (IRB) approval nor patient informed consent was needed or obtained. The study protocol was registered with the International Prospective Register of Systematic Reviews, PROSPERO (registration no: CRD42016039482).

\section{Literature search strategy}

The primary exposure of interest was magnesium supplementation, while the primary outcome of interest was changes in serum C-reactive protein levels subsequent to magnesium supplementation. We searched multiple databases including PubMed/Medline, Cochrane Central Register of Controlled Trials (CCTR), Cochrane Database of Systematic Reviews (CDSR), Web of Science and MEDLINE, until December 2016, using a combination of search terms available in Table I. We used the wild-card term '*' to increase the sensitivity of the search strategy. No language restriction was applied. This was complemented by hand searches of the reference list of eligible articles, and email correspondences with authors for additional data where relevant. All paper abstracts were screened by two reviewers ( $M M$ and $P R$ ) in an initial process to remove ineligible articles. The remaining articles were obtained in full text and assessed again by the same two researchers who evaluated each article independently, carried out data extraction and quality assessment. Disagreements were resolved by discussion with a third party $(\mathrm{HV})$.

\section{Selection criteria}

All prospective studies evaluating the effect of $\mathrm{Mg}$ supplementation on the outcomes of interest were included in this analysis. Eligible studies had to meet the following criteria: (1) prospective controlled trial with either parallel or crossover design and of patients treated with magnesium supplementation compared to a control group (either no

Table I. Full search terms and strategy used for systematically reviewing the articles

\begin{tabular}{|c|c|c|}
\hline No. & Concept & Search terms \\
\hline 1 & Magnesium & ((“magnesium”[Text Word]) OR “Mg”[Text Word]) \\
\hline 2 & C-reactive protein & $\begin{array}{l}\text { "high sensitivity C-reactive protein"[MeSH Terms] OR "high-sensitivity } \\
\text { C-reactive protein"[MeSH Terms] OR “C-reactive protein"[MeSH Terms] OR } \\
\text { "high-sensitive C-reactive protein"[MeSH Terms] OR "high sensitive C-reactive } \\
\text { protein"[MeSH Terms] OR “CRP”[Title/Abstract] OR “hsCRP”[Title/Abstract] }\end{array}$ \\
\hline 3 & Combination & 1 AND 2 \\
\hline
\end{tabular}


Mg supplementation or placebo), (2) presentation of sufficient information on primary outcome at baseline and at the end of follow-up in each group or providing the net change values. Exclusion criteria were: (i) non-clinical studies; (ii) observational studies with case-control, cross-sectional or cohort design; and (iii) studies that did not provide mean (or median) plasma concentrations of our interested outcomes at baseline and/or at the end of trial. Narrative reviews, comments, opinion pieces, methodological, editorials, letters or any other publications lacking primary data and/or explicit method descriptions were also excluded. Study selection started with the removal of duplicates, followed by screening of titles and abstracts by two reviewers. To avoid bias, they were blinded to the names, qualifications or the institutional affiliations of the study authors. The agreement between the reviewers was excellent ( $\kappa$ index: $0.88 ; p<0.001$ ). Disagreements were resolved at a meeting between reviewers prior to selected articles being retrieved (a flow chart is available in Figure 1).

\section{Data extraction and management}

The full text of studies meeting the inclusion criteria was retrieved and screened to determine eligibility by two reviewers (MM, PR). Following assessment of methodological quality, the two reviewers extracted data using a purpose-designed data extraction form and independently summarized what they considered to be the most important results from each study. These summaries were compared and any differences of opinion resolved by discussion and consultation with a third reviewer $(\mathrm{HV})$. Additional necessary calculations on study data were conducted by the first reviewer and checked by the second reviewer. Descriptive data extracted included the first author's name, reference, year of publication, country, design, duration of the study, inclusion criteria, dose, age range, sample size, and male gender (\%).

\section{Quality assessment}

A systematic assessment of bias in the included RCTs was performed using the Cochrane criteria [15]. We sued the following items for the assessment of each study: blinding of participants, allocation concealment, adequacy of random sequence generation, handling of drop-outs (incomplete outcome data), personnel and outcome assessment, selective outcome reporting, as well as any other potential sources of bias. According to the recommendations of the Cochrane Handbook, a judgment of 'yes' indicated low risk of bias, while 'no' indicated a high risk of bias. Labeling an item as 'unclear' indicated an unclear

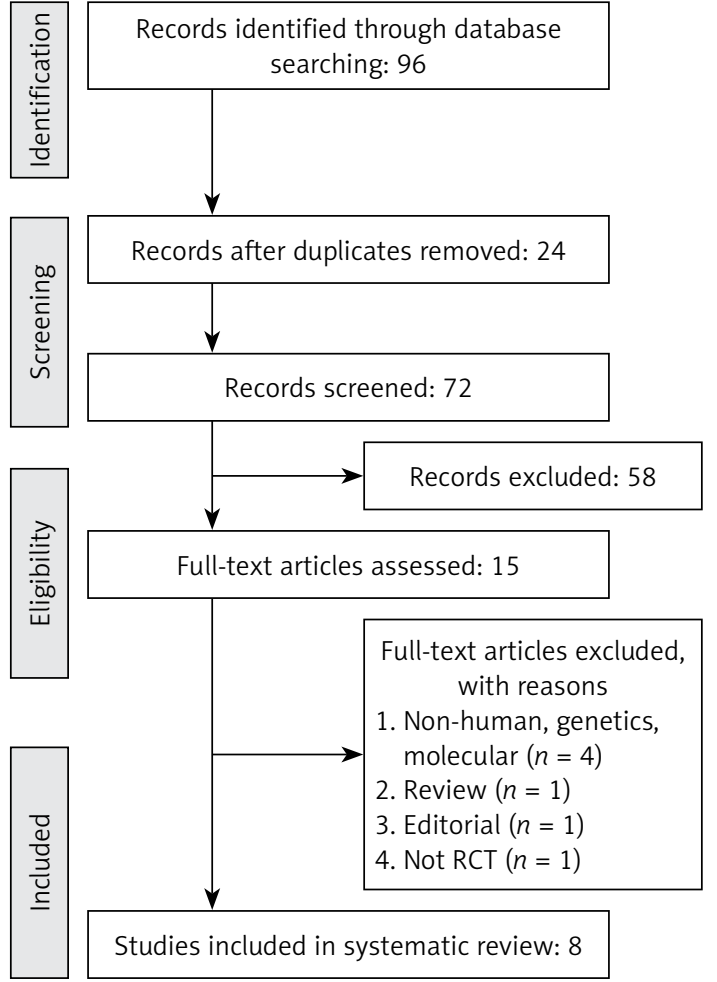

Figure 1. PRISMA flow chart for selection of studies

or unknown risk of bias. Risk-of-bias assessment was performed independently by 2 reviewers (MM and PR); disagreements were resolved by a third reviewer $(\mathrm{HV})$.

\section{Data synthesis}

Following the Cochrane Handbook recommendations for calculating the effect size, we used the mean (SD) change from baseline in the concentrations of the variables of interest for both control and intervention groups [16]. In summary, we calculated the net changes in measurements (change scores) as follows: measure at the end of follow-up - measure at baseline. For RCTs, change scores were calculated as (measure at the end of follow-up in the treatment group - measure at baseline in the treatment group) - (measure at the end of follow-up in the control group - measure at baseline in the control group). In situations where only a standard error of the mean (SEM) was available, we estimated standard deviation (SD) using the following formula: $S D=S E M \times$ square root $(n)$, where $n$ is the number of subjects [17]. In situations where the outcome measures were reported in median and range (or 95\% confidence interval $(\mathrm{Cl})$ ), we estimated the mean (SD) values using the method described by Mazidi et al. [18]. When the outcome variable was available only in the graphic form, the software GetData Graph Digitizer 2.24 [19] was used to digitize and extract the data. Blood glucose level was collated in 
$\mathrm{mmol} / \mathrm{l}$; a multiplication factor of 0.0555 was used to convert glucose levels from $\mathrm{mg} / \mathrm{dl}$ to $\mathrm{mmol} / \mathrm{l}$ as appropriate [20].

A random effects model (using the DerSimonian-Laird method) and the generic inverse variance method were used to compensate for the heterogeneity of studies in terms of demographic characteristics of populations being studied and also differences in study design and type of BAS being studied [21]. Heterogeneity was quantitatively assessed using the $R^{2}$ index. The $R^{2}$ values $<50 \%$ and $\geq 50 \%$ corresponded with the use of fixed-effects and random-effects models, respectively. We expressed the effect sizes as the weighted mean difference (WMD) and 95\% confidence interval (CI). To assess the influence of each study on the overall effect size, we conducted a sensitivity analysis using the leave-one-out method (removing one study each time and repeating the analysis) [22-24].

\section{Meta-regression}

Random-effects meta-regression was performed using the unrestricted maximum likelihood method to evaluate the association between calculated WMD and potential moderator including dose of magnesium supplementation.

\section{Publication bias}

Potential publication bias was explored using visual inspection of Begg's funnel plot asymmetry, Begg's rank correlation and Egger's weighted regression tests. Duval \& Tweedie 'trim and fill' and 'fail-safe N' methods were used to adjust the analysis for the effects of publication bias [25]. Meta-analysis was conducted using Comprehensive Meta-Analysis (CMA) V3 software (Biostat, NJ) [26].

\section{Results}

\section{Summary of searches and study selection process}

A total of 96 unique citations were identified from searches, of which 72 records remained after removing duplicates. After screening via titles and abstracts, 15 articles remained for further evaluation, of which seven were excluded for the following reasons: non-human studies, genetic, or molecular studies $(n=4)$; reviews or editorial articles $(n=2)$ and not RCTs $(n=1)$ (Figure 1$)$. Therefore, eight studies with 349 participants were finally included in the meta-analysis.

\section{Risk of bias assessment}

Several of the included studies were characterized by a lack of information about allocation concealment $(n=1)$ or blinding of outcome assessment $(n=2)$. However, one study had a moderate risk of bias [27] and other evaluated studies had a low risk of bias according to selective outcome reporting. Details of the quality of bias assessment are shown in Table II.

\section{Characteristics of the included studies}

The characteristics of included studies are summarized in Table III. These studies were published between 2004 and 2014 from four countries: the United States of America (5 studies), Mexico (4 studies), Iran and Turkey. The number of participants included in the studies ranged from 14 [28] to 100 [29]. Participants in one study were only female [30], while the proportion of females in five studies ranged from $29 \%$ [8] to $78 \%$ [29]. In two studies the percentage

Table II. Quality of bias assessment of the included studies according to the Cochrane guidelines

\begin{tabular}{|c|c|c|c|c|c|c|c|}
\hline Studies & $\begin{array}{l}\text { Random } \\
\text { sequence } \\
\text { genera- } \\
\text { tion }\end{array}$ & $\begin{array}{c}\text { Allocation } \\
\text { conceal- } \\
\text { ment }\end{array}$ & $\begin{array}{l}\text { Selective } \\
\text { reporting }\end{array}$ & $\begin{array}{l}\text { Blinding } \\
\text { of partici- } \\
\text { pants and } \\
\text { personnel }\end{array}$ & $\begin{array}{l}\text { Blinding } \\
\text { of out- } \\
\text { come as- } \\
\text { sessment }\end{array}$ & $\begin{array}{l}\text { Incom- } \\
\text { plete } \\
\text { outcome } \\
\text { data }\end{array}$ & Other bias \\
\hline Chacko, 2011 [28] & L & $L$ & $\mathrm{~L}$ & L & L & L & $L$ \\
\hline $\begin{array}{l}\text { Simental-Mendía, } \\
2012 \text { [45] }\end{array}$ & L & L & $L$ & L & $U$ & L & $L$ \\
\hline Kazaks, 2010 [32] & L & $\mathrm{L}$ & L & $\mathrm{H}$ & L & L & L \\
\hline Mortazavi, 2012 [46] & $\mathrm{L}$ & $\mathrm{L}$ & $\mathrm{L}$ & L & L & $\mathrm{L}$ & $\mathrm{L}$ \\
\hline Nielsen, 2010 [29] & $\mathrm{L}$ & $U$ & $\mathrm{~L}$ & L & $\mathrm{L}$ & $\mathrm{L}$ & $\mathrm{L}$ \\
\hline Resatoglu, 2004 [27] & $\mathrm{L}$ & $\mathrm{H}$ & $\mathrm{H}$ & L & $U$ & $L$ & L \\
\hline $\begin{array}{l}\text { Rodriguez-Hernandez, } \\
2010 \text { [30] }\end{array}$ & $\mathrm{L}$ & $\mathrm{L}$ & $\mathrm{L}$ & $\mathrm{L}$ & $\mathrm{L}$ & $\mathrm{L}$ & $\mathrm{L}$ \\
\hline $\begin{array}{l}\text { Simental-Mendıa, } \\
2014 \text { [31] }\end{array}$ & $\mathrm{L}$ & $\mathrm{H}$ & $\mathrm{L}$ & L & $\mathrm{L}$ & $\mathrm{L}$ & $\mathrm{L}$ \\
\hline
\end{tabular}




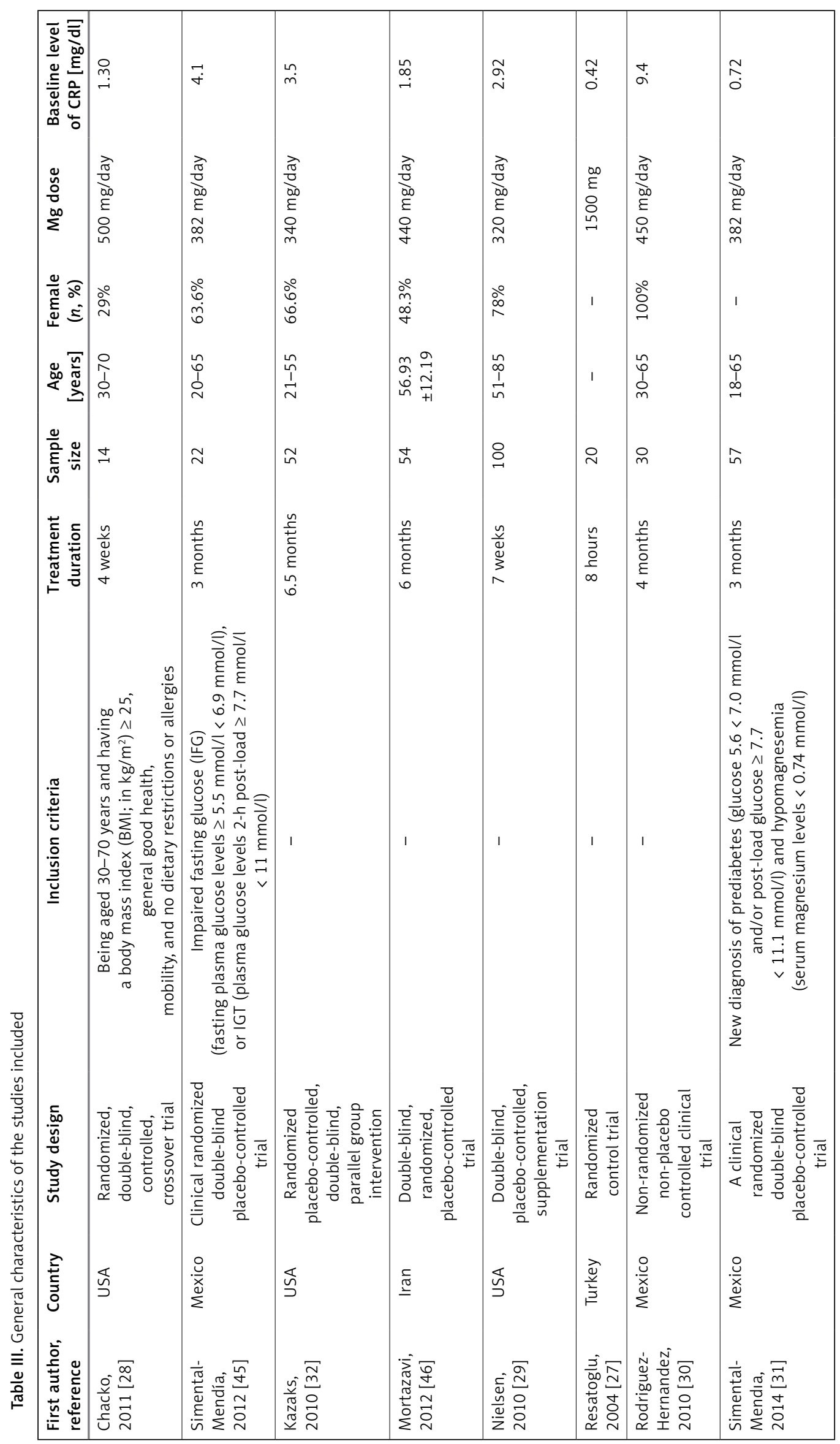


of women was unknown [27, 31]. The mean age of participants ranged from 18 [31] to 85 years [29]. The range of duration of the supplementation intervention across studies was from $8 \mathrm{~h}$ [27] to 6.5 months [32]. The consumed range of $\mathrm{Mg}$ dose in these studies was from 320 [29] to 1500 [27] $\mathrm{mg} /$ day. The baseline level of the CRP varied between the studies from $0.42 \mathrm{mg} /$ dl [6] as minimum to $9.4 \mathrm{mg} / \mathrm{dl}$ [7] as maximum, Table III.

\section{Pooled estimate of the effect of $\mathrm{Mg}$ supplementation on CRP}

The pooled estimate (weighted mean difference) of the effect of $\mathrm{Mg}$ supplementation on CRP levels was $-1.33 \mathrm{mg} / \mathrm{dl}(95 \% \mathrm{Cl}:-2.63$ to -0.02 , $p<0.001$, heterogeneity $p<0.123 ; P^{2}=29.1 \%$ ) across all studies (Figure 2). We divided our studies according to the baseline level of the CRP; it was found that subjects with baseline CRP higher than or equal to 2 (CRP $\geq 2 \mathrm{mg} / \mathrm{dl}$ (4 studies)) have more significant reduction in the CRP level $(-2.95 \mathrm{mg} / \mathrm{dl}$; $95 \% \mathrm{Cl}:-3.35$ to $-2.25, p<0.001$, heterogeneity $\left.p=0.952 ; I^{2}=1.1 \%\right)$ compared with the subjects with CRP $<2 \mathrm{mg} / \mathrm{l}$ (4 studies) $(-0.23$; $95 \% \mathrm{Cl}:-0.195$ to $-0.326, p<0.001$, heterogeneity $p=0.923 ; I^{2}=1.3 \%$ ).

\section{Pooled estimate of the effect of $\mathrm{Mg}$ supplementation on IL-6}

The pooled estimate (weighted mean difference) of the effect of $\mathrm{Mg}$ supplementation on IL-6 levels was $-0.16 \mathrm{pg} / \mathrm{dl}(95 \% \mathrm{Cl}:-3.52$ to $3.26, p=$ 0.236 , heterogeneity $p=0.802 ; I^{2}=2.3 \%$ ) across all studies.

\section{Pooled estimate of the effect of $\mathrm{Mg}$ supplementation on TNF- $\alpha$}

The pooled estimate (weighted mean difference) of the effect of $\mathrm{Mg}$ supplementation on TNF- $\alpha$ levels was $1.97 \mathrm{pg} / \mathrm{dl}(95 \% \mathrm{Cl}: 1.12$ to 2.82 , $p=0.043$, heterogeneity $p=0.869 ; l^{2}=2.1 \%$ ) across all studies.
Pooled estimate of the effect of $\mathrm{Mg}$ supplementation on fasting blood glucose (FBG)

The pooled estimate (weighted mean difference) of the effect of $\mathrm{Mg}$ supplementation on FBG levels was $-0.61 \mathrm{mg} / \mathrm{dl}(95 \% \mathrm{Cl}:-2.72$ to $1.48, p=$ 0.182 , heterogeneity $p=0.742 ; 1^{2}=6.1 \%$ ) across all studies.

Pooled estimate of the effect of $\mathrm{Mg}$ supplementation on systolic blood pressure (SBP)

The pooled estimate (weighted mean difference) of the effect of $\mathrm{Mg}$ supplementation on SBP levels was $-0.93 \mathrm{~mm} \mathrm{Hg}(95 \% \mathrm{Cl}:-3.03$ to 1.20 , $p=0.293$, heterogeneity $p=0.526 ; R^{2}=3.6 \%$ ) across all studies.

\section{Pooled estimate of the effect of $\mathrm{Mg}$ supplementation on diastolic blood pressure (DBP)}

The pooled estimate (weighted mean difference) of the effect of $\mathrm{Mg}$ supplementation on DBP levels was $-0.30 \mathrm{~mm} \mathrm{Hg}(95 \% \mathrm{Cl}:-2.80$ to 2.19 , $p=0.639$, heterogeneity $p=0.489 ; R^{2}=3.8 \%$ ) across all studies.

\section{Pooled estimate of the effect of $\mathrm{Mg}$} supplementation on body mass index (BMI)

The pooled estimate (weighted mean difference) of the effect of $\mathrm{Mg}$ supplementation on BMI levels was $0.27 \mathrm{~kg} / \mathrm{m}^{2}$ (95\% Cl: -0.59 to $1.15, p=$ 0.542 , heterogeneity $p=0.906 ; p^{2}=2.0 \%$ ) across all studies.

\section{Sensitivity analysis}

In leave-one-out sensitivity analyses, the pooled effect estimates remained similar across all studies for CRP $(-1.33 \mathrm{mg} / \mathrm{l} ; 95 \% \mathrm{Cl}:-2.63$ to $-0.02)$. This stability confirms that the significant difference between the studied groups is the overall effect of all included studies.

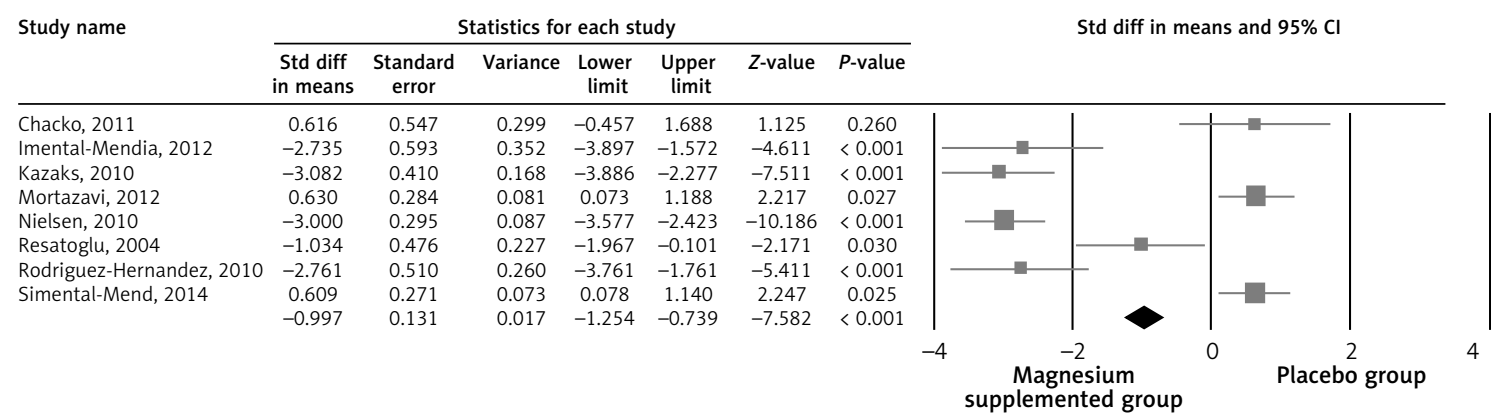

Figure 2. Forest plot displaying weighted mean difference and $95 \%$ confidence intervals for the impact of magnesium supplementation on CRP levels 


\section{Meta regression}

Random-effects meta-regression was performed to evaluate the impact of potential moderators on the estimated effect size. Changes in plasma CRP levels were independent of the dosage of $\mathrm{Mg}$ (slope: $-0.004 ; 95 \% \mathrm{Cl}$ : $-0.03,0.02$; $p=0.720$, Figure 3 ) and duration (slope: -0.06 ; $95 \% \mathrm{Cl}:-0.37,0.24 ; p=0.681$, Figure 4 ) of supplementation.

\section{Publication bias}

Visual inspection of funnel plot asymmetry suggested potential publication bias for the comparison of plasma CRP levels between Mg supplemented groups and placebo groups (Figure 5); however, the presence of publication bias was not suggested by Egger's linear regression (intercept $=-5.69$, standard error $=6.10 ; 95 \% \mathrm{Cl}:-20.64$, 9.25, $t=0.93, \mathrm{~d} f=6.00$, two-tailed $p=0.387$ ) and Begg's rank correlation test (Kendall's Tau with continuity correction $=-0.14, z=0.49$, two tailed $p=0.620$ ) was not indicative for publication bias. After adjustment of the effect size for potential publication bias using the 'trim and fill' correction, no potentially missing studies were imputed in the funnel plot (WMD $-1.33 \mathrm{mg} / \mathrm{l} ; 95 \% \mathrm{Cl}:-2.63$ to -0.02 , Figure 6). The 'fail-safe N' test showed that 146 studies would be needed to bring the WMD down to a non-significant $(p>0.05)$ value.

\section{Discussion}

The present meta-analysis of 8 RCTs published in the last 10 years (2004-2014) showed that $\mathrm{Mg}$ supplementation significantly decreased the level of serum hs-CRP by $-1.33 \mathrm{mg} / \mathrm{l}(-2.63$ to -0.02$)$. Evidence from this meta-analysis indicates that dietary $\mathrm{Mg}$ intake is inversely associated with serum CRP levels. Our results showed that Mg supplementation did not have a significant effect on IL-6 $(-0.16 \mathrm{pg} / \mathrm{dl})$. These results support the hypothesis that dietary $\mathrm{Mg}$ plays a beneficial role in the regu-

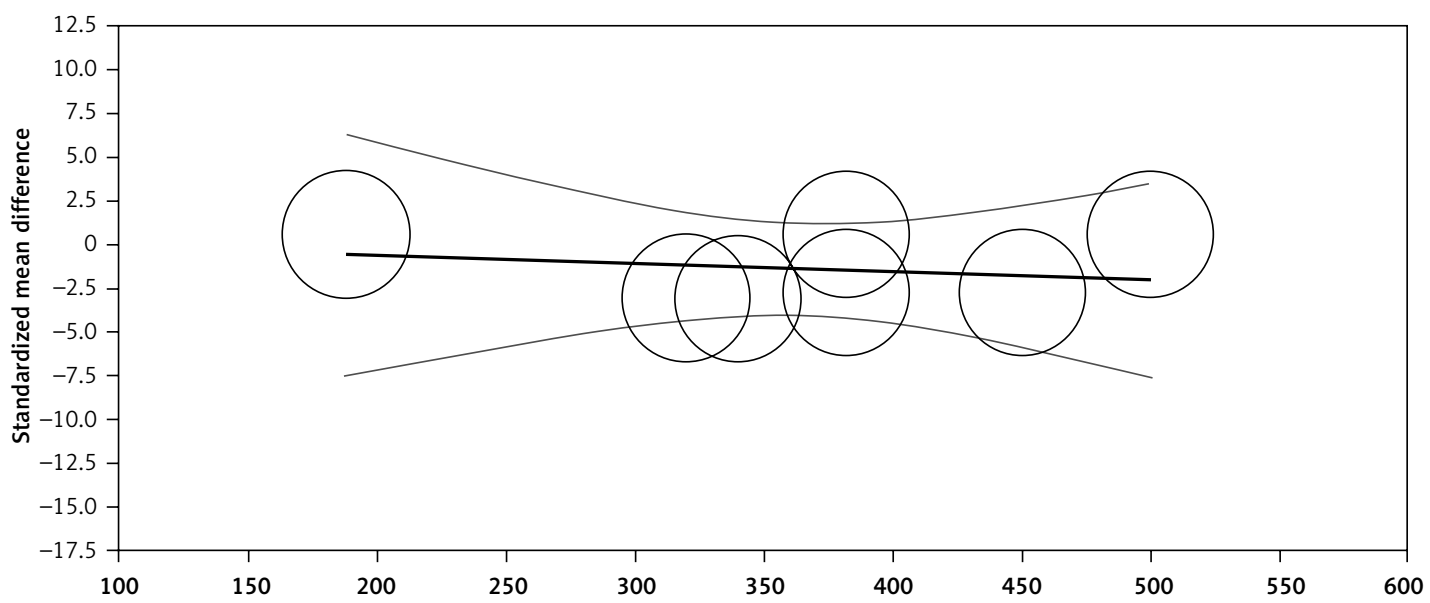

Figure 3. Meta-regression plots of the association between mean changes in CRP levels with doses of magnesium supplementation. Circles represent each study, middle line is the regression line, two lines around the middle line represent the $95 \%$ confidence interval

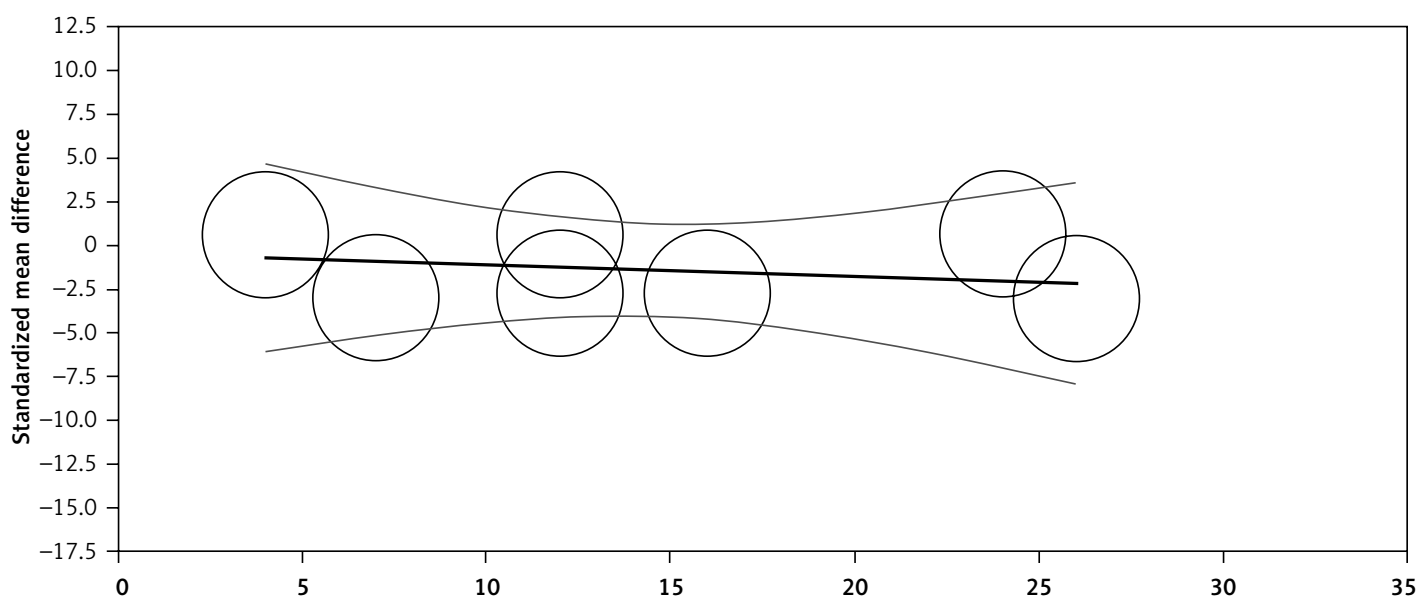

Figure 4. Meta-regression plots of the association between mean changes in CRP levels with duration of magnesium supplementation. Circles represent each study, middle line is the regression line, two lines around the middle line represent the $95 \%$ confidence interval 


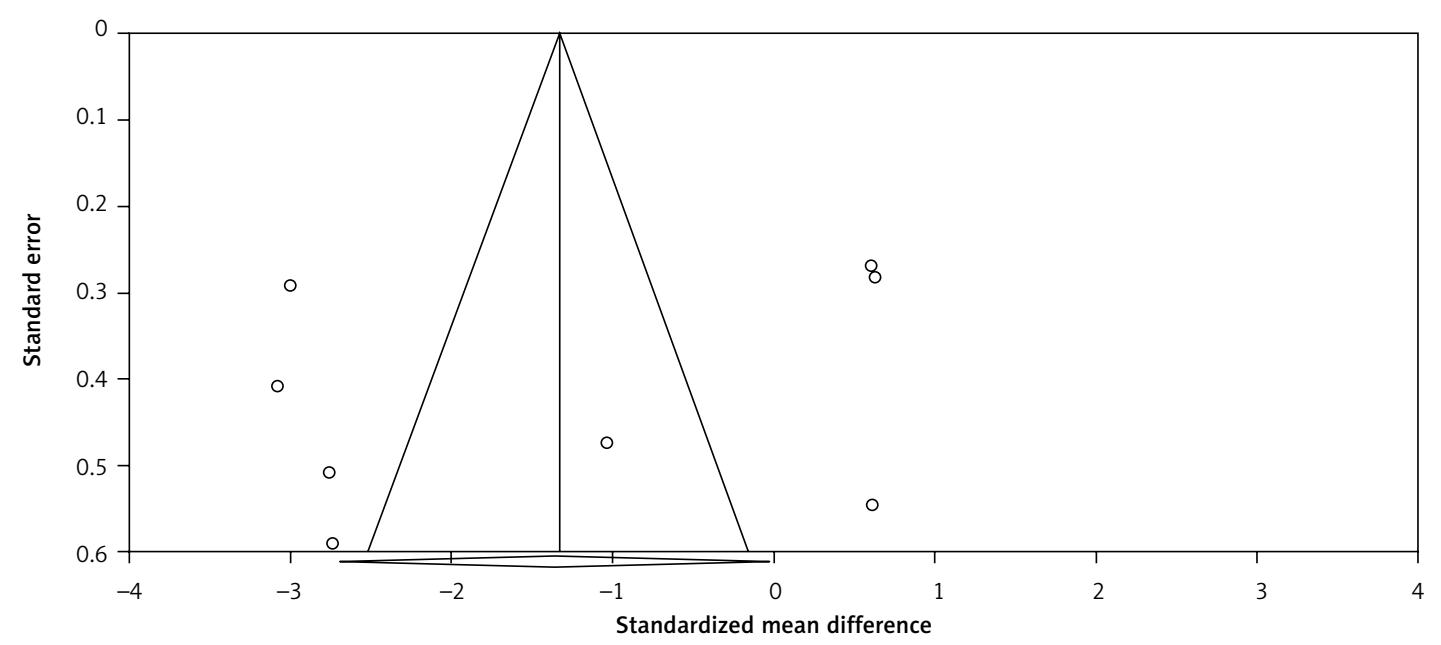

Figure 5. Funnel plots detailing publication bias in the studies selected for analysis. Open diamond represents observed effect size; open circles represent observed published studies

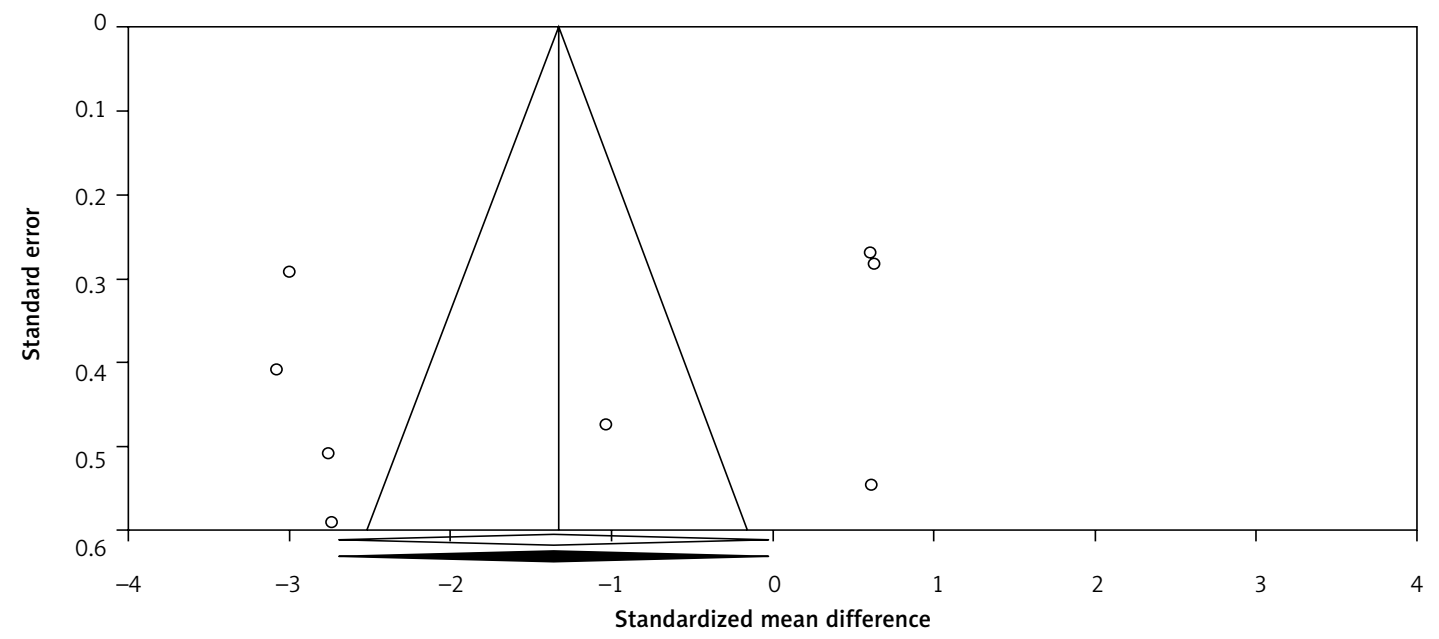

Figure 6. Trim and fill method was used to impute for potentially missing studies. No potentially missing study was imputed in funnel plot, open circles represent observed published studies; open diamond represents observed effect size; closed diamond represents imputed effect size

lation of inflammatory markers. In this regard, King [33] recently surveyed data of several cross-sectional and epidemiological studies highlighting that $\mathrm{Mg}$ possibly plays an important role in potentiating inflammatory processes. The inverse association found in our meta-analysis is supported by evidence from randomized controlled studies that have reported on patients with type 2 diabetes, admitted to an intensive care unit, and among patients with heart failure, oral Mg supplementation decreased CRP levels [34, 35]. However, previous data published regarding the efficacy of oral $\mathrm{Mg}$ supplementation for reducing CRP levels are scarce [35]. Regarding effects of Mg intake on IL-6 level, some studies have found inconsistent results, while others have shown an inverse association [36], but others did not demonstrate any correlation [37]. IL-6, which acts as both a pro-inflammatory and an anti-inflammatory cytokine, secreted by $T$ cells and macrophages, is the major mediator of fever and the acute phase response [38]. As raised IL-6 concentrations may be an early indicator of acute inflammation, increased serum IL- 6 concentrations may be observed because of undetected underlying acute infection present simultaneously with $\mathrm{Mg}$ usage by chance [35]. Although the Mg-related primary events that produce the acute-phase response are not known with certainty, this complex process suggests that $\mathrm{Mg}$ deficiency might be among the initial elements that trigger the inflammatory response [39]. Evidence derived from an animal model have shown that acute $\mathrm{Mg}$ deficiency leads to an inflammatory response [40]. Moreover, human studies show that low serum $\mathrm{Mg}$ levels are strongly associated with raised CRP concentration $[3,41]$. A potential mechanism for the association between $\mathrm{Mg}$ deficiency and inflammation is related to calcium. If dietary $\mathrm{Mg}$ intake is inadequate, it may deplete extracellular Mg ions and consequently cause stimulation of macrophages as well as an 
influx of calcium ions into cells such as adipocytes, neuronal and peritoneal cells. N-methyl-D-aspartate receptors (NMDARs) are glutamate-gated cation channels with high calcium permeability. Augmented calcium levels in the cells enhanced $\mathrm{Mg}$ necessary to block the influx of calcium ions, which further led to increased stimulation of these receptors. Stimulation of these NMDAR results in the opening of channels nonselective to cations, consequently increasing calcium ions in neuronal cells [42]. This change causes the release of neurotransmitters (e.g., substance P) and inflammatory cytokines. IL- 6 is released into the bloodstream and acts as a signaling molecule to enhance the release of CRP from the liver as a step in the acute phase response, which promotes prolongation of the inflammatory response in the body [1].

There are some potential limitations in our analysis that need to be addressed. Firstly, the majority of the included studies had relatively small sample sizes, potentially leading to unstable estimates of treatment effects, because smaller trials might be methodologically less robust and are prone to report larger effect sizes [43-46]. Moreover, due to the fact that the population at the baseline was very heterogeneous, we expect that it could also accept the output of the metaanalysis; however, to decrease the chance of bias based on the baseline level of the variables we performed a meta-regression and explored the impact of the dose and duration of the supplementation. Lastly, the number of available studies concerning the described topic was rather small, particularly in the case of TNF- $\alpha$, which may lead to misinterpretation of the results.

In conclusion, this meta-analysis suggests that Mg supplementation significantly decreased serum CRP, especially with the baseline values $\geq 2 \mathrm{mg} / \mathrm{dl}$. To provide more conclusive results and clarify the mechanistic pathways, RCTs with a larger sample size and a long-term follow-up period are warranted.

\section{Acknowledgments}

Trial registration: Systematic review registration: CRD42016039482.

MM was supported by a TWAS studentship of the Chinese Academy of Sciences, during the preparation of this manuscript.

\section{Conflict of interest}

The authors declare no conflict of interest.

\section{References}

1. Liu S, Chacko SA. Dietary Mg intake and biomarkers of inflammation and endothelial dysfunction. Magnesium in Human Health and Disease. Springer 2013; 35-50.
2. Song Y, Sesso HD, Manson JE, Cook NR, Buring JE, Liu S. Dietary magnesium intake and risk of incident hypertension among middle-aged and older US women in a 10year follow-up study. Am J Cardiol 2006; 98: 1616-21.

3. Song Y, Ridker PM, Manson JE, Cook NR, Buring JE, Liu S. Magnesium intake, C-reactive protein, and the prevalence of metabolic syndrome in middle-aged and older US women. Diabetes Care 2005; 28: 1438-44.

4. He K, Liu K, Daviglus ML, et al. Magnesium intake and incidence of metabolic syndrome among young adults. Circulation 2006; 113: 1675-82.

5. Paolisso G, Barbagallo M. Hypertension, diabetes mellitus, and insulin resistance: the role of intracellular magnesium. Am J Hypertens 1997; 10: 346-55.

6. Stevanović S, Nikolić M, Stanković A. Dietary magnesium intake and coronary heart disease risk: a study from Serbia. Medicinski Glasnik 2011; 8: 203-8.

7. Guerrera MP, Volpe SL, Mao JJ. Therapeutic uses of magnesium. Am Fam Physician 2009; 80: 157-62.

8. Guerrero-Romero F, Rascón-Pacheco R, Rodríguez-Morán $M$, La Peña D, Escobedo J, Wacher N. Hypomagnesaemia and risk for metabolic glucose disorders: a 10-year follow-up study. Eur J Clin Investig 2008; 38: 389-96.

9. Kramer JH, Mak IT, Tejero-Taldo MI, et al. Neurogenic inflammation and cardiac dysfunction due to hypomagnesemia. Am J Med Sci 2009; 338: 22-7.

10. Mazidi M, Kengne AP, Mikhailidis DP, Cicero AF, Banach $M$. Effects of selected dietary constituents on high-sensitivity C-reactive protein levels in U.S. adults. Ann Med 2018; 50: 1-6.

11. King DE, Mainous III AG, Geesey ME, Woolson RF. Dietary magnesium and C-reactive protein levels. J Am Coll Nutr 2005; 24: 166-71.

12. de Oliveira Otto MC, Alonso A, Lee DH, et al. Dietary micronutrient intakes are associated with markers of inflammation but not with markers of subclinical atherosclerosis. J Nutr 2011; 141: 1508-15.

13. Moher D, Liberati A, Tetzlaff J, Altman DG. Preferred reporting items for systematic reviews and meta-analyses: the PRISMA statement. Ann Intern Med 2009; 151: 264-9.

14. Phan K, Tian DH, Cao C, Black D, Yan TD. Systematic review and meta-analysis: techniques and a guide for the academic surgeon. Ann Cardiothorac Surg 2015; 4: 112-22.

15. Higgins JPT GSe. Cochrane Handbook for Systematic Reviews of Interventions. Version 5.0.2. London: The Cochrane Collaboration 2009

16. Higgins J, Green S. Cochrane handbook for systematic reviews, version 5.0. 2 The Cochrane Collaboration. OpenURL; 2009.

17. Mazidi M, Rezaie P, Gao HK, Kengne AP. Effect of sodium-glucose cotransport-2 inhibitors on blood pressure in people with type 2 diabetes mellitus: a systematic review and meta-analysis of 43 randomized control trials with 22528 patients. J Am Heart Assoc 2017; 6: e004007.

18. Hozo SP, Djulbegovic B, Hozo I. Estimating the mean and variance from the median, range, and the size of a sample. BMC Med Res Methodol 2005; 5: 13.

19. Mazidi M, Karimi E, Rezaie P, Ferns GA. Effects of conjugated linoleic acid supplementation on serum C-reactive protein: a systematic review and meta-analysis of randomized controlled trials. Cardiovasc Ther 2017; 35: e12275.

20. Mazidi M, Rezaie P, Karimi E, Kengne AP. The effects of bile acid sequestrants on lipid profile and blood glucose concentrations: a systematic review and meta-analysis 
of randomized controlled trials. Int J Cardiol 2017; 227: 850-7.

21. Mazidi M, Gao HK, Rezaie P, Ferns GA. The effect of ginger supplementation on serum C-reactive protein, lipid profile and glycaemia: a systematic review and metaanalysis. Food Nutr Res 2016; 60: 32613.

22. Mazidi M, Karimi E, Rezaie P, Ferns GA. Treatment with GLP1 receptor agonists reduce serum CRP concentrations in patients with type 2 diabetes mellitus: a systematic review and meta-analysis of randomized controlled trials. J Diabetes Complications 2017; 31: 1237-42.

23. Mazidi M, Rezaie P, Vatanparast H, Kengne AP. Effect of statins on serum vitamin $D$ concentrations: a systematic review and meta-analysis. Eur J Clin Invest 2017; 47: 93-101.

24. Mazidi M, Rezaie P, Ferns GA, Gao HK. Impact of different types of tree nut, peanut, and soy nut consumption on serum C-reactive protein (CRP): a systematic review and meta-analysis of randomized controlled clinical trials. Medicine (Baltimore) 2016; 95: e5165.

25. Duval S, Tweedie R. Trim and fill: a simple funnel-plotbased method of testing and adjusting for publication bias in meta-analysis. Biometrics 2000; 56: 455-63.

26. Borenstein M, Hedges L, Higgins J, Rothstein H. Comprehensive Metaanalysis (Vers. 2). Englewood Cliffs, NJ: Biostat. Inc; 2005.

27. Resatoglu AG, Demirturk O, Yener N, Yener A. Magnesium decreases cardiac injury in patients undergoing coronary artery bypass surgery. Ann Saudi Med 2004; 24: 259-61.

28. Chacko SA, Sul J, Song Y, et al. Magnesium supplementation, metabolic and inflammatory markers, and global genomic and proteomic profiling: a randomized, double-blind, controlled, crossover trial in overweight individuals. Am J Clin Nutr 2011; 93: 463-73.

29. Nielsen FH, Johnson LK, Zeng H. Magnesium supplementation improves indicators of low magnesium status and inflammatory stress in adults older than 51 years with poor quality sleep. Magnes Res 2010; 23: 158-68.

30. Rodriguez-Hernandez H, Cervantes-Huerta M, Rodriguez-Moran M, Guerrero-Romero F. Oral magnesium supplementation decreases alanine aminotransferase levels in obese women. Magnes Res 2010; 23: 90-6.

31. Simental-Mendía LE, Rodríguez-Morán M, GuerreroRomero F. Oral magnesium supplementation decreases C-reactive protein levels in subjects with prediabetes and hypomagnesemia: a clinical randomized double-blind placebo-controlled trial. Arch Med Res 2014; 45: 325-30.

32. Kazaks AG, Uriu-Adams JY, Albertson TE, Shenoy SF Stern JS. Effect of oral magnesium supplementation on measures of airway resistance and subjective assessment of asthma control and quality of life in men and women with mild to moderate asthma: a randomized placebo controlled trial. J Asthma 2010; 47: 83-92.

33. King DE. Inflammation and elevation of C-reactive protein: does magnesium play a key role? Magnes Res 2009; 22: 57-9.

34. Curiel-García JA, Rodríguez-Morán M, Guerrero-Romero F. Hypomagnesemia and mortality in patients with type 2 diabetes. Magnes Res 2008; 21: 163-6.

35. Almoznino-Sarafian D, Berman S, Mor A, et al. Magnesium and C-reactive protein in heart failure: an anti-inflammatory effect of magnesium administration? Eur J Nutr 2007; 46: 230-7.

36. Song Y, Li TY, van Dam RM, Manson JE, Hu FB. Magnesium intake and plasma concentrations of markers of systemic inflammation and endothelial dysfunction in women. Am J Clin Nutr 2007; 85: 1068-74.

37. Chacko SA, Song Y, Nathan L, et al. Relations of dietary magnesium intake to biomarkers of inflammation and endothelial dysfunction in an ethnically diverse cohort of postmenopausal women. Diabetes Care 2010; 33: 304-10.

38. Epstein FH, Gabay C, Kushner I. Acute-phase proteins and other systemic responses to inflammation. N Engl J Med 1999; 340: 448-54.

39. Malpuech-Brugère C, Rock E, Astier C, Nowacki W, Mazur A, Rayssiguier Y. Exacerbated immune stress response during experimental magnesium deficiency results from abnormal cell calcium homeostasis. Life Sci 1998; 63: 1815-22.

40. Malpuech-Brugère $C$, Nowacki W, Daveau $M$, et al. Inflammatory response following acute magnesium deficiency in the rat. Biochim Biophys Acta Mol Basis Dis 2000; 1501: 91-8.

41. Guerrero-Romero F, Rodríguez-Morán M. Hypomagnesemia, oxidative stress, inflammation, and metabolic syndrome. Diabetes Metabol Res Rev 2006; 22: 471-6.

42. Nielsen FH. Magnesium, inflammation, and obesity in chronic disease. Nutr Rev 2010; 68: 333-40.

43. Nuesch E, Trelle S, Reichenbach S, et al. Small study effects in meta-analyses of osteoarthritis trials: meta-epidemiological study. BMJ 2010; 341: c3515.

44. Sterne JA, Gavaghan D, Egger M. Publication and related bias in meta-analysis: power of statistical tests and prevalence in the literature. J Clin Epidemiol 2000; 53 : 1119-29.

45. Simental-Mendía LE, Rodríguez-Morán M, Reyes-Romero MA, Guerrero-Romero F. No positive effect of ora magnesium supplementation in the decreases of in flammation in subjects with prediabetes: a pilot study. Magnes Res 2012; 25: 140-6.

46. Mortazavi M, Moeinzadeh F, Saadatnia M, Shahidi S, McGee JC, Minagar A. Effect of magnesium supplementation on carotid intima-media thickness and flow-mediated dilatation among hemodialysis patients: a double-blind, randomized, placebo-controlled trial. Eur Neurol 2013; 69: 309-16. 